Unfallchirurg 2015 $\cdot 118: 650-651$

DOI 10.1007/s00113-015-0043-6

Online publiziert: 2. Juli 2015

c) Springer-Verlag Berlin Heidelberg 2015

Gerrit Matthes ${ }^{1}$. Sascha Flohé2 Thomas Paffrath $^{3} \cdot$ Heiko Trentzsch ${ }^{4}$. Christoph Wölfl' ${ }^{5}$

${ }^{1}$ Unfallkrankenhaus Berlin, Klinik für Unfallchirurgie und Orthopädie, Berlin, Deutschland

2 Städtisches Klinikum Solingen, Unfallchirurgie, Orthopädie \& Handchirurgie, Solingen, Deutschland

${ }^{3}$ Krankenhaus Köln-Merheim, Orthopädie, Unfallchirurgie \& Sportmedizin, Köln-Merheim, Deutschland

${ }^{4}$ Institut für Notfallmedizin und Medizinmanagement (INM),

Klinikum der Universität München, München, Deutschland

${ }^{5}$ BGU Ludwigshafen, Unfallchirurgie \& Orthopädie, Ludwigshafen, Deutschland

\title{
Die initiale Versorgung des schwerverletzten Patienten
}

\section{Eine unfallchirurgische Herausforderung}

Die Versorgung schwerverletzter Patienten ist Kernkompetenz der Unfallchirurgie. Sie beginnt mit der präklinischen notärztlichen Versorgung, umfasst die komplette klinische Behandlung und endet schließlich mit der Rehabilitation.

Die Sektion Notfallmedizin, Intensivmedizin und Schwerverletztenversorgung (NIS) der Deutschen Gesellschaft für Unfallchirurgie e. V. beschäftigt sich schwerpunktmäßig mit diesem Themenkreis. Die Autoren der Artikel dieses Themenheftes rekrutieren sich aus dem aktiven Mitgliederkreis.

Verbesserungen der medizinischen Versorgung in den letzten Jahrzehnten haben einen deutlichen Rückgang der Todesfälle nach schwerer Verletzung bedingt. Es bedarf allerdings weiterer Anstrengungen, die derzeitigen Versorgungsstrategien zu verbessern. Trotz eines auch im internationalen Vergleich vorbildlich ausgestalteten notärztlichen Versorgungskonzeptes kommt es immer noch zu vermeidbaren Todesfällen noch vor Erreichen der Zielklinik. Hier gilt es, klare Vorgaben zu machen, welche therapeutischen Maßnahmen bereits präklinisch zwingend durchzuführen sind. Im intensivmedizinischen Umfeld haben sich in den letzten Jahren so genannte "care bundles“ erfolgreich etabliert, welche evidenzbasierte, einfach umsetzbare Maßnahmen definieren, die in einer spezifischen Situation umgesetzt werden müssen.
In einem Artikel von G. Matthes et al. wird ein „care bundle“ zur präklinischen Versorgung des schwerverletzten Patienten vorgestellt. Nach Versorgung am Unfallort müssen verletzte Patienten zügig mit dem geeigneten Rettungsmittel in die richtige Klinik verbracht werden. Auch hier ist es unabdingbar, entsprechende Strukturen zu definieren. Das Traumanetzwerk D hat dabei wichtige Dienste leisten können.

In einer Arbeit von A. Paul et al. wird ein Tool vorgestellt, das es ermöglicht, Schwachstellen innerhalb der Schnittstelle Präklinik/Klinik eines Traumanetzwerks zu identifizieren und auf diese Weise im Rahmen der Qualitätssicherung gezielte Schritte zur Verbesserung aufzuzeigen.

Mit Übergabe des Patienten müssen dann umgehend die richtige Diagnostik und Versorgung eingeleitet werden. Eine Arbeit von S. Piatek et al. beschäftigt sich in diesem Kontext mit der Wertigkeit klinischer Leitsymptome.

Sowohl der Unfallort als auch der Schockraum stellen Arbeitsbereiche dar, in denen ein hohes Fehlerrisiko besteht. Eine entsprechende Fehlerkultur ist daher zu fordern. In einem Übersichtsartikel von $H$. Trentzsch et al. werden mögliche Fehler definiert und Lösungsansätze aufgezeigt.

Wir freuen uns über die spannenden Beiträge dieses Themenhefts und hoffen, dass sie auch bei der Leserschaft auf ein breites Interesse stoßen.

An dieser Stelle möchten wir interessierte Kolleginnen und Kollegen ermun- tern, in der Sektion NIS aktiv mitzuarbei-

ten.

Ihre

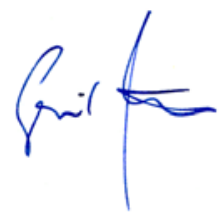

Gerrit Matthes

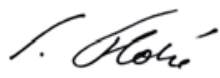

Sascha Flohé

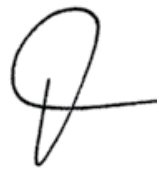

Thomas Paffrath

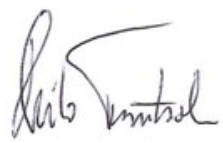

Heiko Trentzsch

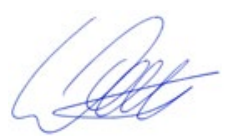

Christoph Wölfl 


Korrespondenzadresse
Unfallkrankenhaus Berlin
Klinik für Unfallchirurgie und
Orthopädie, Warenerstr. 7
12683 Berlin
gerrit.matthes@ukb.de

\section{Einhaltung ethischer Richtlinien}

Interessenkonflikt. G. Matthes, S. Flohé, T. Paffrath, H. Trentzsch und C. Wölfl geben an, dass kein Interessenkonflikt besteht.

\section{Lesetipp}

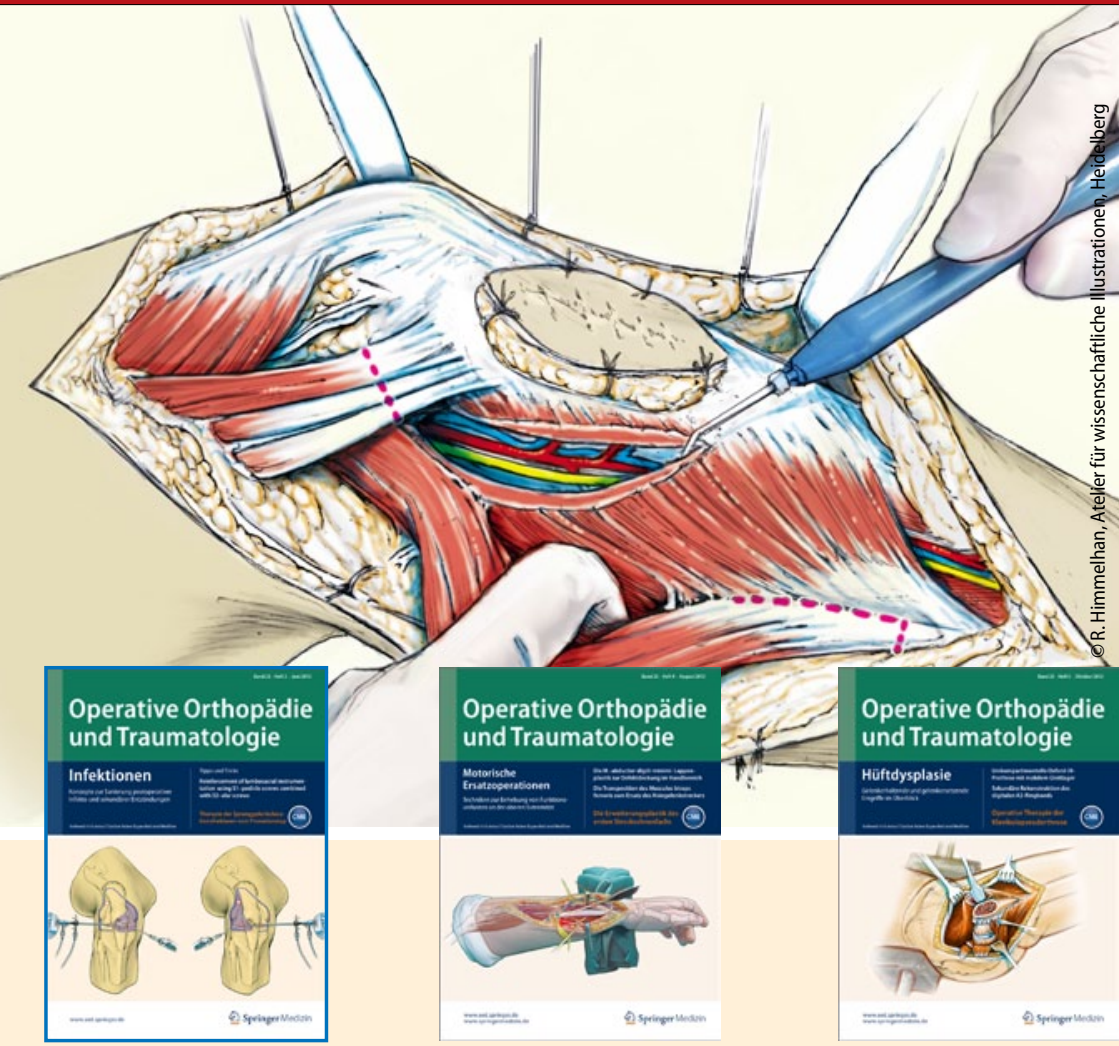

Die Zeitschrift Operative Orthopädie und Traumatologie bietet Ihnen in 6 Ausgaben pro Jahr fundierte Informationen zu bewährten und neuen Operationstechniken der Orthopädie, Unfallchirurgie und Wiederherstellungschirurgie. In den aufwendig illustrierten Beiträgen werden die Operationsverfahren Schritt für Schritt vorgestellt.

Möchten Sie ein bereits erschienenes Heft nachbestellen? Einzelne Ausgaben können Sie direkt bei unserem Kundenservice zum Preis von je EUR 73,- zzgl. Versandkosten beziehen:

\section{Heft 1/2015: Leitthema „Periprothetische Defekte am Knie“}

- Defektrekonstruktion in der Knieendoprothetik mit Wedges und Blöcken

- Defektrekonstruktion beim Knieendoprothesenwechsel mit Tantal-Konen

- Revisionsendoprothetik des Kniegelenks

- Impaction bone grafting for reconstruction of large bone defekts in revision knee arthroplasty

- CME: Motorische Ersatzplastik durch Sehnentransposition bei Lähmung des Nervus radialis

Heft 2/2015: Leitthema „Operative Rekonstruktionsverfahren beim Charcot-Fuß“

- Behandlung von Rückfußdestruktionen beim Charcot-Fuß durch Hybridtechnik mit interner Osteosynthese und Ringfixateur

- Tibiotalokalkaneare Korrekturarthrodese bei Charcot-Arthropathie des OSG mit dem retrograden Nagel

- Behandlung von Fußwurzelinstabilitäten bei Charcot-Fuß mit dem Ringfixateur

- CME: Naht und Refixation des medialen Kollateralbandkomplexes bei Instabilität des Kniegelenks

So erreichen Sie unseren Kundenservice:

Springer Customer Service Center GmbH Kundenservice Zeitschriften

Haberstr. 7

69126 Heidelberg

Tel.: +496221 345-4303

Fax: +496221 345-4229

E-Mail: leserservice@springer.com

www.oot.springer.de 